\title{
Foundations of generalized Prabhakar-Hilfer fractional calculus with applications
}

\author{
George A. Anastassiou ${ }^{1}$ \\ ${ }^{1}$ Department of Mathematical Sciences \\ University of Memphis, Memphis, \\ TN 38152, U.S.A. \\ ganastss@memphis.edu
}

\begin{abstract}
Here we introduce the generalized Prabhakar fractional calculus and we also combine it with the generalized Hilfer calculus. We prove that the generalized left and right side Prabhakar fractional integrals preserve continuity and we find tight upper bounds for them. We present several left and right side generalized Prabhakar fractional inequalities of Hardy, Opial and Hilbert-Pachpatte types. We apply these in the setting of generalized Hilfer calculus.
\end{abstract}

\section{RESUMEN}

Introducimos el cálculo fraccionario generalizado de Prabhakar y también lo combinamos con el cálculo generalizado de Hilfer. Demostramos que las integrales fraccionarias generalizadas de Prabhakar izquierda y derecha preservan la continuidad y encontramos cotas superiores ajustadas para ellas. Presentamos diversas desigualdades fraccionarias generalizadas de Prabhakar izquierda y derecha de tipos Hardy, Opial y Hilbert-Pachpatte. Aplicamos estos resultados en el contexto del cálculo generalizado de Hilfer.

Keywords and Phrases: Prabhakar fractional calculus, Hilfer fractional calculus, fractional Hardy, Opial and Hilbert-Pachpatte inequalities.

2020 AMS Mathematics Subject Classification: 26A33, 26D10, 26 D15. 


\section{Background}

During the last 50 years fractional calculus due to its wide applications to many applied sciences has become a main trend in mathematics. Its predominant kinds are the old Riemann-Liouville fractional calculus and the newer one of Caputo type. Around these two versions have been built a plethora of other variants and all of these involve singular kernels. More recently researchers presented also new fractional calculi of non singular kernels.

The recent Hilfer fractional calculus unifies the Riemann-Liouville and Caputo fractional calculi and the Prabhakar fractional calculus unifies both singular and non-singular kernel fractional calculi.

Finally the newer Hilfer-Prabhakar fractional calculus is the most general one unifying all trends and for different values of its parameters we get the particular fractional calculi. In this article we present and employ unifying advanced and generalized versions of Prabhakar and Hilfer-Prabhakar fractional calculi and we establish related unifying fractional integral inequalities of the following types: Hardy, Opial and Hilbert-Pachpatte. The advantage of this unification is the uniform action taken in describing the various natural phenomena.

We are inspired by [7], [6] and [1]. We start by introducing our own generalized $\psi$-Prabhakar type of fractional calculus, then mixing it with the $\psi$-Hilfer fractional calculus. Then, we prove a variety of generalized Hardy, Opial and Hilbert-Pachpatte type left and right fractional integral inequalities related to $\psi$-Hilfer ([8]) and $\psi$-Prabhakar fractional calculi. We involve several functions.

We consider the Prabhakar function (also known as the three parameter Mittag-Leffler function), (see [4, p. 97]; [3])

$$
E_{\alpha, \beta}^{\gamma}(z)=\sum_{k=0}^{\infty} \frac{(\gamma)_{k}}{k ! \Gamma(\alpha k+\beta)} z^{k}
$$

where $\Gamma$ is the gamma function; $\alpha, \beta, \gamma \in \mathbb{R}: \alpha, \beta>0, z \in \mathbb{R}$, and $(\gamma)_{k}=\gamma(\gamma+1) \cdots(\gamma+k-1)$. It is $E_{\alpha, \beta}^{0}(z)=\frac{1}{\Gamma(\beta)}$.

Let $a, b \in \mathbb{R}, a<b$ and $x \in[a, b] ; f \in C([a, b])$. Let also $\psi \in C^{1}([a, b])$ which is increasing. The left and right Prabhakar fractional integrals with respect to $\psi$ are defined as follows:

$$
\left(e_{\rho, \mu, \omega, a+}^{\gamma ; \psi} f\right)(x)=\int_{a}^{x} \psi^{\prime}(t)(\psi(x)-\psi(t))^{\mu-1} E_{\rho, \mu}^{\gamma}\left[\omega(\psi(x)-\psi(t))^{\rho}\right] f(t) d t,
$$

and

$$
\left(e_{\rho, \mu, \omega, b-}^{\gamma ; \psi} f\right)(x)=\int_{x}^{b} \psi^{\prime}(t)(\psi(t)-\psi(x))^{\mu-1} E_{\rho, \mu}^{\gamma}\left[\omega(\psi(t)-\psi(x))^{\rho}\right] f(t) d t,
$$

where $\rho, \mu>0 ; \gamma, \omega \in \mathbb{R}$.

Functions (1.2) and (1.3) are continuous, see Theorem 3.1.

Next, additionally, assume that $\psi^{\prime}(x) \neq 0$ over $[a, b]$.

Let $\psi, f \in C^{N}([a, b])$, where $N=\lceil\mu\rceil,(\lceil\cdot\rceil$ is the ceiling of the number $), 0<\mu \notin \mathbb{N}$. We define the 
$\psi$-Prabhakar-Caputo left and right fractional derivatives of order $\mu$ as follows $(x \in[a, b])$ :

$$
\begin{gathered}
\left({ }^{C} D_{\rho, \mu, \omega, a+}^{\gamma ; \psi} f\right)(x)=\int_{a}^{x} \psi^{\prime}(t)(\psi(x)-\psi(t))^{N-\mu-1} \\
E_{\rho, N-\mu}^{-\gamma}\left[\omega(\psi(x)-\psi(t))^{\rho}\right]\left(\frac{1}{\psi^{\prime}(t)} \frac{d}{d t}\right)^{N} f(t) d t
\end{gathered}
$$

and

$$
\begin{gathered}
\left({ }^{C} D_{\rho, \mu, \omega, b-}^{\gamma ; \psi} f\right)(x)=(-1)^{N} \int_{x}^{b} \psi^{\prime}(t)(\psi(t)-\psi(x))^{N-\mu-1} \\
E_{\rho, N-\mu}^{-\gamma}\left[\omega(\psi(t)-\psi(x))^{\rho}\right]\left(\frac{1}{\psi^{\prime}(t)} \frac{d}{d t}\right)^{N} f(t) d t
\end{gathered}
$$

One can write (see (1.4), (1.5))

$$
\left({ }^{C} D_{\rho, \mu, \omega, a+}^{\gamma ; \psi} f\right)(x)=\left(e_{\rho, N-\mu, \omega, a+}^{-\gamma ; \psi} f_{\psi}^{[N]}\right)(x),
$$

and

$$
\left({ }^{C} D_{\rho, \mu, \omega, b-}^{\gamma ; \psi} f\right)(x)=(-1)^{N}\left(e_{\rho, N-\mu, \omega, b-}^{-\gamma ; \psi} f_{\psi}^{[N]}\right)(x),
$$

where

$$
f_{\psi}^{[N]}(x)=f_{\psi}^{(N)} f(x):=\left(\frac{1}{\psi^{\prime}(x)} \frac{d}{d x}\right)^{N} f(x)
$$

$\forall x \in[a, b]$.

Functions (1.6) and (1.7) are continuous on $[a, b]$.

Next we define the $\psi$-Prabhakar-Riemann-Liouville left and right fractional derivatives of order $\mu$ as follows $(x \in[a, b])$ :

$$
\begin{gathered}
\left({ }^{R L} D_{\rho, \mu, \omega, a+}^{\gamma ; \psi} f\right)(x)=\left(\frac{1}{\psi^{\prime}(x)} \frac{d}{d x}\right)^{N} \int_{a}^{x} \psi^{\prime}(t)(\psi(x)-\psi(t))^{N-\mu-1} \\
E_{\rho, N-\mu}^{-\gamma}\left[\omega(\psi(x)-\psi(t))^{\rho}\right] f(t) d t
\end{gathered}
$$

and

$$
\begin{gathered}
\left({ }^{R L} D_{\rho, \mu, \omega, b-}^{\gamma ; \psi} f\right)(x)=\left(-\frac{1}{\psi^{\prime}(x)} \frac{d}{d x}\right)^{N} \int_{x}^{b} \psi^{\prime}(t)(\psi(t)-\psi(x))^{N-\mu-1} \\
E_{\rho, N-\mu}^{-\gamma}\left[\omega(\psi(t)-\psi(x))^{\rho}\right] f(t) d t .
\end{gathered}
$$

That is we have

$$
\left({ }^{R L} D_{\rho, \mu, \omega, a+}^{\gamma ; \psi} f\right)(x)=\left(\frac{1}{\psi^{\prime}(x)} \frac{d}{d x}\right)^{N}\left(e_{\rho, N-\mu, \omega, a+}^{-\gamma ; \psi} f\right)(x)
$$

and

$$
\left({ }^{R L} D_{\rho, \mu, \omega, b-}^{\gamma ; \psi} f\right)(x)=\left(-\frac{1}{\psi^{\prime}(x)} \frac{d}{d x}\right)^{N}\left(e_{\rho, N-\mu, \omega, b-}^{-\gamma ; \psi} f\right)(x)
$$

$\forall x \in[a, b]$. 
We define also the $\psi$-Hilfer-Prabhakar left and right fractional derivatives of order $\mu$ and type $0 \leq \beta \leq 1$, as follows

$$
\left({ }^{H} \mathbb{D}_{\rho, \mu, \omega, a+}^{\gamma, \beta ; \psi} f\right)(x)=e_{\rho, \beta(N-\mu), \omega, a+}^{-\gamma \beta ; \psi}\left(\frac{1}{\psi^{\prime}(x)} \frac{d}{d x}\right)^{N} e_{\rho,(1-\beta)(N-\mu), \omega, a+}^{-\gamma(1-\beta) \psi} f(x),
$$

and

$$
\left({ }^{H} \mathbb{D}_{\rho, \mu, \omega, b-}^{\gamma, \beta ; \psi} f\right)(x)=e_{\rho, \beta(N-\mu), \omega, b-}^{-\gamma \beta ; \psi}\left(-\frac{1}{\psi^{\prime}(x)} \frac{d}{d x}\right)^{N} e_{\rho,(1-\beta)(N-\mu), \omega, b-}^{-\gamma(1-\beta) ; \psi} f(x),
$$

$\forall x \in[a, b]$.

When $\beta=0$, we get the Riemann-Liouville version, and when $\beta=1$, we get the Caputo version.

We call $\xi=\mu+\beta(N-\mu)$, we have that $N-1<\mu \leq \mu+\beta(N-\mu) \leq \mu+N-\mu=N$, hence $\lceil\xi\rceil=N$.

We can easily write that

$$
\left({ }^{H} \mathbb{D}_{\rho, \mu, \omega, a+}^{\gamma, \beta ; \psi} f\right)(x)=e_{\rho, \xi-\mu, \omega, a+}^{-\gamma \beta ; \psi}{ }^{R L} D_{\rho, \xi, \omega, a+}^{\gamma(1-\beta) ; \psi} f(x),
$$

and

$$
\left({ }^{H} \mathbb{D}_{\rho, \mu, \omega, b-}^{\gamma, \beta ; \psi} f\right)(x)=e_{\rho, \xi-\mu, \omega, b-}^{-\gamma \beta ; \psi}{ }^{R L} D_{\rho, \xi, \omega, b-}^{\gamma(1-\beta) ; \psi} f(x),
$$

$\forall x \in[a, b]$.

\section{Main results}

We start with a left $\psi$-Prabhakar fractional Hardy type integral inequality involving several functions.

Theorem 2.1. Here $i=1, \ldots, m ; f_{i} \in C([a, b]), \psi \in C^{1}([a, b])$ and $\psi$ is increasing. Let $\rho_{i}, \mu_{i}>0$, $\gamma_{i}, \omega_{i} \in \mathbb{R}$. Also let $r_{1}, r_{2}, r_{3}>1: \frac{1}{r_{1}}+\frac{1}{r_{2}}+\frac{1}{r_{3}}=1$, and assume that $\mu_{i}>\frac{1}{r_{2}}+\frac{1}{r_{3}}$, for all $i=1, \ldots, m$.

Then

$$
\begin{aligned}
& \left\|\prod_{i=1}^{m} e_{\rho_{i}, \mu_{i}, \omega_{i}, a+}^{\gamma_{i} ; \psi} f_{i}\right\|_{L_{r_{1}([a, b], \psi)}} \leq \\
& (\psi(b)-\psi(a))^{\left[\sum_{i=1}^{m} \mu_{i}-m+\frac{m}{r_{1}}+\frac{1}{r_{1}}-\frac{1}{r_{1} r_{2}}\right]} \\
& \overline{\left(r_{1} r_{3}\left(\sum_{i=1}^{m} \mu_{i}-m\right)+m r_{3}+1\right)^{\frac{1}{r_{1} r_{3}}}\left(\prod_{i=1}^{m}\left(r_{1}\left(\mu_{i}-1\right)+1\right)\right)^{\frac{1}{r_{1}}}} \\
& \left\{\int_{a}^{b}\left[\prod_{i=1}^{m}\left(\int_{a}^{x}\left|E_{\rho_{i}, \mu_{i}}^{\gamma_{i}}\left[\omega_{i}(\psi(x)-\psi(t))^{\rho_{i}}\right]\right|^{r_{2}} d \psi(t)\right)\right]^{r_{1}} d \psi(x)\right\}^{\frac{1}{r_{1} r_{2}}} \\
& \left(\prod_{i=1}^{m}\left\|f_{i}\right\|_{L_{r_{3}}([a, b], \psi)}\right)
\end{aligned}
$$


Proof. By (1.2) we have

$$
\left(e_{\rho_{i}, \mu_{i}, \omega_{i}, a+}^{\gamma_{i} ; \psi} f_{i}\right)(x)=\int_{a}^{x} \psi^{\prime}(t)(\psi(x)-\psi(t))^{\mu_{i}-1} E_{\rho_{i}, \mu_{i}}^{\gamma_{i}}\left[\omega_{i}(\psi(x)-\psi(t))^{\rho_{i}}\right] f_{i}(t) d t,
$$

$i=1, \ldots, m ; \forall x \in[a, b]$.

By Hölder's inequality and (2.2) we obtain

$$
\begin{gathered}
\left|\left(e_{\rho_{i}, \mu_{i}, \omega_{i}, a+}^{\gamma_{i}, \psi} f_{i}\right)(x)\right| \leq \\
\int_{a}^{x} \psi^{\prime}(t)(\psi(x)-\psi(t))^{\mu_{i}-1}\left|E_{\rho_{i}, \mu_{i}}^{\gamma_{i}}\left[\omega_{i}(\psi(x)-\psi(t))^{\rho_{i}}\right]\right|\left|f_{i}(t)\right| d t \leq \\
\left(\int_{a}^{x}(\psi(x)-\psi(t))^{r_{1}\left(\mu_{i}-1\right)} d \psi(t)\right)^{\frac{1}{r_{1}}} \\
\left(\int_{a}^{x}\left|E_{\rho_{i}, \mu_{i}}^{\gamma_{i}}\left[\omega_{i}(\psi(x)-\psi(t))^{\rho_{i}}\right]\right|^{r_{2}} d \psi(t)\right)^{\frac{1}{r_{2}}}\left(\int_{a}^{x}\left|f_{i}(t)\right|^{r_{3}} d \psi(t)\right)^{\frac{1}{r_{3}}} \leq \\
\frac{(\psi(x)-\psi(a))^{\mu_{i}-1+\frac{1}{r_{1}}}}{\left(r_{1}\left(\mu_{i}-1\right)+1\right)^{\frac{1}{r_{1}}}} \\
\left(\int_{a}^{x}\left|E_{\rho_{i}, \mu_{i}}^{\gamma_{i}}\left[\omega_{i}(\psi(x)-\psi(t))^{\rho_{i}}\right]\right|^{r_{2}} d \psi(t)\right)^{\frac{1}{r_{2}}}\left\|f_{i}\right\|_{L_{r_{3}}([a, b], \psi)} .
\end{gathered}
$$

So far we have

$$
\begin{gathered}
\left|\left(e_{\rho_{i}, \mu_{i}, \omega_{i}, a+}^{\gamma_{i} ; f_{i}}\right)(x)\right| \leq \frac{(\psi(x)-\psi(a))^{\mu_{i}-1+\frac{1}{r_{1}}}}{\left(r_{1}\left(\mu_{i}-1\right)+1\right)^{\frac{1}{r_{1}}}} \\
\left(\int_{a}^{x}\left|E_{\rho_{i}, \mu_{i}}^{\gamma_{i}}\left[\omega_{i}(\psi(x)-\psi(t))^{\rho_{i}}\right]\right|^{r_{2}} d \psi(t)\right)^{\frac{1}{r_{2}}}\left\|f_{i}\right\|_{L_{r_{3}}([a, b], \psi)},
\end{gathered}
$$

$\forall x \in[a, b]$, with $\mu_{i}>\frac{1}{r_{2}}+\frac{1}{r_{3}}$, for any $i=1, \ldots, m$.

Hence it holds

$$
\begin{gathered}
\left(\prod_{i=1}^{m}\left|\left(e_{\rho_{i}, \mu_{i}, \omega_{i}, a+}^{\gamma_{i} ;} f_{i}\right)(x)\right|\right)^{r_{1}} \leq \frac{(\psi(x)-\psi(a))^{r_{1} \sum_{i=1}^{m} \mu_{i}-m r_{1}+m}}{\left(\prod_{i=1}^{m}\left(r_{1}\left(\mu_{i}-1\right)+1\right)\right)} \\
{\left[\prod_{i=1}^{m}\left(\int_{a}^{x}\left|E_{\rho_{i}, \mu_{i}}^{\gamma_{i}}\left[\omega_{i}(\psi(x)-\psi(t))^{\rho_{i}}\right]\right|^{r_{2}} d \psi(t)\right)\right]^{\frac{r_{1}}{r_{2}}}\left(\prod_{i=1}^{m}\left\|f_{i}\right\|_{L_{r_{3}}([a, b], \psi)}\right)^{r_{1}},}
\end{gathered}
$$

$\forall x \in[a, b]$.

Therefore we obtain

$$
\begin{gathered}
\int_{a}^{b}\left(\prod_{i=1}^{m}\left|\left(e_{\rho_{i}, \mu_{i}, \omega_{i}, a+}^{\gamma_{i} ;} f_{i}\right)(x)\right|\right)^{r_{1}} d \psi(x) \leq \frac{\left(\prod_{i=1}^{m}\left\|f_{i}\right\|_{L_{r_{3}}([a, b], \psi)}\right)^{r_{1}}}{\left(\prod_{i=1}^{m}\left(r_{1}\left(\mu_{i}-1\right)+1\right)\right)} \\
{\left[\int_{a}^{b}(\psi(x)-\psi(a))^{r_{1} \sum_{i=1}^{m} \mu_{i}-m r_{1}+m}\right.}
\end{gathered}
$$




$$
\left.\left[\prod_{i=1}^{m}\left(\int_{a}^{x}\left|E_{\rho_{i}, \mu_{i}}^{\gamma_{i}}\left[\omega_{i}(\psi(x)-\psi(t))^{\rho_{i}}\right]\right|^{r_{2}} d \psi(t)\right)\right]^{\frac{r_{1}}{r_{2}}} d \psi(x)\right]
$$

(again by Hölder's inequality)

$$
\begin{gathered}
\left.\leq \frac{\left(\prod_{i=1}^{m}\left\|f_{i}\right\|_{L_{r_{3}}([a, b], \psi)}\right)^{r_{1}}\left(\prod_{i=1}^{m}\left(r_{1}\left(\mu_{i}-1\right)+1\right)\right)}{b}(\psi(x)-\psi(a))^{r_{1} r_{3} \sum_{i=1}^{m} \mu_{i}-m r_{1} r_{3}+m r_{3}} d \psi(x)\right)^{\frac{1}{r_{3}}} \\
\left\{\int_{a}^{b}\left[\prod_{i=1}^{m}\left(\int_{a}^{x}\left|E_{\rho_{i}, \mu_{i}}^{\gamma_{i}}\left[\omega_{i}(\psi(x)-\psi(t))^{\rho_{i}}\right]\right|^{r_{2}} d \psi(t)\right)\right]^{r_{1}} d \psi(x)\right\}^{\frac{1}{r_{2}}}(\psi(b)-\psi(a))^{\frac{1}{r_{1}}}= \\
\frac{\left(\prod_{i=1}^{m}\left\|f_{i}\right\|_{L_{r_{3}}([a, b], \psi)}\right)^{r_{1}}(\psi(b)-\psi(a))^{r_{1} \sum_{i=1}^{m} \mu_{i}-m r_{1}+m+1-\frac{1}{r_{2}}}}{\left(\prod_{i=1}^{m}\left(r_{1}\left(\mu_{i}-1\right)+1\right)\right)\left(r_{1} r_{3} \sum_{i=1}^{m} \mu_{i}-m r_{1} r_{3}+m r_{3}+1\right)^{\frac{1}{r_{3}}}} \\
\left\{\int_{a}^{b}\left[\prod_{i=1}^{m}\left(\int_{a}^{x}\left|E_{\rho_{i}, \mu_{i}}^{\gamma_{i}}\left[\omega_{i}(\psi(x)-\psi(t))^{\rho_{i}}\right]\right|^{r_{2}} d \psi(t)\right)\right]^{r_{1}} d \psi(x)\right\}^{\frac{1}{r_{2}}},
\end{gathered}
$$

where $\mu_{i}>\frac{1}{r_{2}}+\frac{1}{r_{3}}, i=1, \ldots, m$.

The claim is proved.

We continue with a right $\psi$-Prabhakar fractional Hardy type integral inequality involving several functions.

Theorem 2.2. All as in Theorem 2.1. It holds

$$
\begin{gathered}
\left\|\prod_{i=1}^{m} e_{\rho_{i}, \mu_{i}, \omega_{i}, b-}^{\gamma_{i} ; \psi} f_{i}\right\|_{L_{r_{1}}([a, b], \psi)} \leq \\
\frac{(\psi(b)-\psi(a))^{\left[\sum_{i=1}^{m} \mu_{i}-m+\frac{m}{r_{1}}+\frac{1}{r_{1}}-\frac{1}{r_{1} r_{2}}\right]}}{\left(r_{1} r_{3}\left(\sum_{i=1}^{m} \mu_{i}-m\right)+m r_{3}+1\right)^{\frac{1}{r_{1} r_{3}}}\left(\prod_{i=1}^{m}\left(r_{1}\left(\mu_{i}-1\right)+1\right)\right)^{\frac{1}{r_{1}}}} \\
\left\{\int_{a}^{b}\left[\prod_{i=1}^{m}\left(\int_{x}^{b}\left|E_{\rho_{i}, \mu_{i}}^{\gamma_{i}}\left[\omega_{i}(\psi(t)-\psi(x))^{\rho_{i}}\right]\right|^{r_{2}} d \psi(t)\right)\right]^{r_{1}} d \psi(x)\right\}^{\frac{1}{r_{1} r_{2}}} \\
\left(\prod_{i=1}^{m}\left\|f_{i}\right\|_{L_{r_{3}}([a, b], \psi)}\right) .
\end{gathered}
$$

Proof. Similar to the proof of Theorem 2.1 and omitted.

Next we apply Theorems 2.1, 2.2.

We give the related Hardy type inequalities: 
Theorem 2.3. Here $i=1, \ldots, m ; f_{i} \in C^{N_{i}}([a, b])$, where $N_{i}=\left\lceil\mu_{i}\right\rceil, 0<\mu_{i} \notin \mathbb{N} ; \theta:=$ $\max \left\{N_{1}, \ldots, N_{m}\right\}, \psi \in C^{\theta}([a, b])$ with $\psi^{\prime} \neq 0$ and increasing. Let $\rho_{i}>0, \gamma_{i}, \omega_{i} \in \mathbb{R}$. Also let $r_{1}, r_{2}, r_{3}>1: \frac{1}{r_{1}}+\frac{1}{r_{2}}+\frac{1}{r_{3}}=1$, and assume that $N_{i}-\mu_{i}>\frac{1}{r_{2}}+\frac{1}{r_{3}}$, for all $i=1, \ldots, m$. Then

i)

$$
\begin{gathered}
\left\|\prod_{i=1}^{m}{ }^{C} D_{\rho_{i}, \mu_{i}, \omega_{i}, a+}^{\gamma_{i}, \psi} f_{i}\right\|_{L_{r_{1}}([a, b], \psi)} \leq \\
\frac{(\psi(b)-\psi(a))\left[\sum_{i=1}^{m}\left(N_{i}-\mu_{i}\right)-m+\frac{m}{r_{1}}+\frac{1}{r_{1}}-\frac{1}{r_{1} r_{2}}\right]}{\left(r_{1} r_{3}\left(\sum_{i=1}^{m}\left(N_{i}-\mu_{i}\right)-m\right)+m r_{3}+1\right)^{\frac{1}{r_{1} r_{3}}}\left(\prod_{i=1}^{m}\left(r_{1}\left(N_{i}-\mu_{i}-1\right)+1\right)\right)^{\frac{1}{r_{1}}}} \\
\left\{\int_{a}^{b}\left[\prod_{i=1}^{m}\left(\int_{a}^{x}\left|E_{\rho_{i}, N_{i}-\mu_{i}}^{-\gamma_{i}}\left[\omega_{i}(\psi(x)-\psi(t))^{\rho_{i}}\right]\right|^{r_{2}} d \psi(t)\right)\right]^{r_{1}} d \psi(x)\right\}^{\frac{1}{r_{1} r_{2}}} \\
\left(\prod_{i=1}^{m}\left\|f_{i \psi}^{\left[N_{i}\right]}\right\|_{L_{r_{3}}([a, b], \psi)}\right)
\end{gathered}
$$

and

ii)

$$
\begin{gathered}
\left\|\prod_{i=1}^{m}{ }^{C} D_{\rho_{i}, \mu_{i}, \omega_{i}, b-}^{\gamma_{i} ; \psi} f_{i}\right\|_{L_{r_{1}}([a, b], \psi)} \leq \\
\frac{(\psi(b)-\psi(a))^{\left[\sum_{i=1}^{m}\left(N_{i}-\mu_{i}\right)-m+\frac{m}{r_{1}}+\frac{1}{r_{1}}-\frac{1}{r_{1} r_{2}}\right]}}{\left(r_{1} r_{3}\left(\sum_{i=1}^{m}\left(N_{i}-\mu_{i}\right)-m\right)+m r_{3}+1\right)^{\frac{1}{r_{1} r_{3}}}\left(\prod_{i=1}^{m}\left(r_{1}\left(N_{i}-\mu_{i}-1\right)+1\right)\right)^{\frac{1}{r_{1}}}} \\
\left\{\int_{a}^{b}\left[\prod_{i=1}^{m}\left(\int_{x}^{b}\left|E_{\rho_{i}, N_{i}-\mu_{i}}^{-\gamma_{i}}\left[\omega_{i}(\psi(t)-\psi(x))^{\rho_{i}}\right]\right|^{r_{2}} d \psi(t)\right)\right]^{r_{1}} d \psi(x)\right\}^{\frac{1}{r_{1} r_{2}}} \\
\left(\prod_{i=1}^{m}\left\|f_{i \psi}^{\left[N_{i}\right]}\right\|_{L_{r_{3}}([a, b], \psi)}\right)
\end{gathered}
$$

Proof. By (1.6), (1.7) and Theorems 2.1, 2.2.

We also present other Hardy type related inequalities:

Theorem 2.4. Here $i=1, \ldots, m ; f_{i} \in C^{N_{i}}([a, b])$, where $N_{i}=\left\lceil\mu_{i}\right\rceil, 0<\mu_{i} \notin \mathbb{N} ; \theta:=$ $\max \left\{N_{1}, \ldots, N_{m}\right\}, \psi \in C^{\theta}([a, b]), \psi^{\prime} \neq 0$, and $\psi$ is increasing. Let $\rho_{i}>0, \gamma_{i}, \omega_{i} \in \mathbb{R}, 0 \leq \beta_{i} \leq 1$, $\xi_{i}=\mu_{i}+\beta_{i}\left(N_{i}-\mu_{i}\right)$. Also let $r_{1}, r_{2}, r_{3}>1: \frac{1}{r_{1}}+\frac{1}{r_{2}}+\frac{1}{r_{3}}=1$, and assume that $\xi_{i}-\mu_{i}>\frac{1}{r_{2}}+\frac{1}{r_{3}}$, for all $i=1, \ldots, m$.

Also assume that ${ }^{R L} D_{\rho_{i}, \xi_{i}, \omega_{i}, a+}^{\gamma_{i}\left(1-\beta_{i}\right) ; \psi} f_{i},{ }^{R L} D_{\rho_{i}, \xi_{i}, \omega_{i}, b-}^{\gamma_{i}\left(1-\beta_{i}\right) ; \psi} f_{i} \in C([a, b]), i=1, \ldots, m$.

Then 
i)

$$
\begin{gathered}
\left\|\prod_{i=1}^{m} H_{\mathbb{D}_{\rho_{i}, \mu_{i}, \omega_{i}, a+}^{\gamma_{i}, \beta_{i} ; \psi} f_{i}}\right\|_{L_{r_{1}}([a, b], \psi)} \leq \\
\frac{(\psi(b)-\psi(a))^{\left[\sum_{i=1}^{m}\left(\xi_{i}-\mu_{i}\right)-m+\frac{m}{r_{1}}+\frac{1}{r_{1}}-\frac{1}{r_{1} r_{2}}\right]}}{\left(r_{1} r_{3}\left(\sum_{i=1}^{m}\left(\xi_{i}-\mu_{i}\right)-m\right)+m r_{3}+1\right)^{\frac{1}{r_{1} r_{3}}}\left(\prod_{i=1}^{m}\left(r_{1}\left(\xi_{i}-\mu_{i}-1\right)+1\right)\right)^{\frac{1}{r_{1}}}} \\
\left\{\int_{a}^{b}\left[\prod_{i=1}^{m}\left(\int_{a}^{x}\left|E_{\rho_{i}, \xi_{i}-\mu_{i}}^{-\gamma_{i} \beta_{i}}\left[\omega_{i}(\psi(x)-\psi(t))^{\rho_{i}}\right]\right|^{r_{2}} d \psi(t)\right)\right]^{r_{1}} d \psi(x)\right\}^{\frac{1}{r_{1} r_{2}}} \\
\left(\prod_{i=1}^{m}\left\|R L D_{\rho_{i}, \xi_{i}, \omega_{i}, a+}^{\gamma_{i}\left(1, \beta_{i}\right) ;} f_{i}\right\|_{L_{r_{3}}([a, b], \psi)}\right)
\end{gathered}
$$

and

ii)

$$
\begin{gathered}
\left\|\prod_{i=1}^{m} H_{\mathbb{D}_{\rho_{i}, \mu_{i}, \omega_{i}, b-}^{\gamma_{i}, \beta_{i} ; \psi} f_{i}}\right\|_{L_{r_{1}}([a, b], \psi)} \leq \\
\frac{(\psi(b)-\psi(a))^{\left[\sum_{i=1}^{m}\left(\xi_{i}-\mu_{i}\right)-m+\frac{m}{r_{1}}+\frac{1}{r_{1}}-\frac{1}{r_{1} r_{2}}\right]}}{\left(r_{1} r_{3}\left(\sum_{i=1}^{m}\left(\xi_{i}-\mu_{i}\right)-m\right)+m r_{3}+1\right)^{\frac{1}{r_{1} r_{3}}}\left(\prod_{i=1}^{m}\left(r_{1}\left(\xi_{i}-\mu_{i}-1\right)+1\right)\right)^{\frac{1}{r_{1}}}} \\
\left\{\int_{a}^{b}\left[\prod_{i=1}^{m}\left(\int_{x}^{b}\left|E_{\rho_{i}, \xi_{i}-\mu_{i}}^{-\gamma_{i} \beta_{i}}\left[\omega_{i}(\psi(t)-\psi(x))^{\rho_{i}}\right]\right|^{r_{2}} d \psi(t)\right)\right]^{r_{1}} d \psi(x)\right\}^{\frac{1}{r_{1} r_{2}}} \\
\left(\prod_{i=1}^{m} \| R L D_{\rho_{i}, \xi_{i}, \omega_{i}, b-f_{i}}^{\gamma_{i}\left(1-\beta_{i}\right) ; \|_{r_{3}}([a, b], \psi)}\right)
\end{gathered}
$$

Proof. By (1.15), (1.16) and Theorems 2.1, 2.2.

From now on all entities are according and respectively to Section 1. Background.

Next we give Opial type inequalities related to Prabhakar fractional calculus.

A left side one follows:

Theorem 2.5. Let $p, q>1: \frac{1}{p}+\frac{1}{q}=1$. Then

$$
\begin{gathered}
\int_{a}^{x}\left|\left(e_{\rho, \mu, \omega, a+}^{\gamma ; \psi} f\right)(w)\right||f(w)| \psi^{\prime}(w) d w \leq 2^{-\frac{1}{q}} \\
{\left[\int_{a}^{x}\left\{\int_{a}^{w}(\psi(w)-\psi(t))^{p(\mu-1)}\left|E_{\rho, \mu}^{\gamma}\left[\omega(\psi(w)-\psi(t))^{\rho}\right]\right|^{p} d t\right\} d w\right]^{\frac{1}{p}}} \\
\left(\int_{a}^{x}|f(w)|^{q}\left(\psi^{\prime}(w)\right)^{q} d w\right)^{\frac{2}{q}}
\end{gathered}
$$

$\forall x \in[a, b]$. 
Proof. By (1.2), using Hölder's inequality, we have

$$
\begin{gathered}
\left|\left(e_{\rho, \mu, \omega, a+}^{\gamma ; \psi} f\right)(x)\right| \leq \int_{a}^{x} \psi^{\prime}(t)(\psi(x)-\psi(t))^{\mu-1}\left|E_{\rho, \mu}^{\gamma}\left[\omega(\psi(x)-\psi(t))^{\rho}\right]\right||f(t)| d t \\
\leq\left(\int_{a}^{x}(\psi(x)-\psi(t))^{p(\mu-1)}\left|E_{\rho, \mu}^{\gamma}\left[\omega(\psi(x)-\psi(t))^{\rho}\right]\right|^{p} d t\right)^{\frac{1}{p}} \\
\left(\int_{a}^{x}\left(\psi^{\prime}(t)|f(t)|\right)^{q} d t\right)^{\frac{1}{q}} .
\end{gathered}
$$

Call

$$
\phi(x)=\int_{a}^{x}\left(\psi^{\prime}(t)|f(t)|\right)^{q} d t, \quad \phi(a)=0 .
$$

Thus

$$
\phi^{\prime}(x)=\left(\psi^{\prime}(x)|f(x)|\right)^{q} \geq 0,
$$

and

$$
\left(\phi^{\prime}(x)\right)^{\frac{1}{q}}=\psi^{\prime}(x)|f(x)| \geq 0, \quad \forall x \in[a, b] .
$$

Consequently, we get

$$
\begin{gathered}
\left|\left(e_{\rho, \mu, \omega, a+}^{\gamma ; \psi} f\right)(w)\right| \psi^{\prime}(w)|f(w)| \leq \\
\left(\int_{a}^{w}(\psi(w)-\psi(t))^{p(\mu-1)}\left|E_{\rho, \mu}^{\gamma}\left[\omega(\psi(w)-\psi(t))^{\rho}\right]\right|^{p} d t\right)^{\frac{1}{p}} \\
\left(\phi(w) \phi^{\prime}(w)\right)^{\frac{1}{q}}, \quad \forall w \in[a, b] .
\end{gathered}
$$

Then, by applying again Hölder's inequality:

$$
\begin{gathered}
\int_{a}^{x}\left|\left(e_{\rho, \mu, \omega, a+}^{\gamma ; \psi}\right)(w)\right||f(w)| \psi^{\prime}(w) d w \leq \\
\int_{a}^{x}\left\{\int_{a}^{w}(\psi(w)-\psi(t))^{p(\mu-1)}\left|E_{\rho, \mu}^{\gamma}\left[\omega(\psi(w)-\psi(t))^{\rho}\right]\right|^{p} d t\right\}^{\frac{1}{p}} \\
{\left[\int _ { a } ^ { x } \left\{\int_{a}^{w}\left(\psi(w) \phi^{\prime}(w)\right)^{\frac{1}{q}} d w \leq\right.\right.} \\
\left(\int_{a}^{x} \phi(w) d \phi(w)\right)^{\frac{1}{q}}= \\
\left.\left[\left.\int_{a}^{x}\left\{\int_{a}^{w}(\psi(w)-\psi(t))^{p(\mu-1)}\left|E_{\rho, \mu}^{\gamma}\left[\omega(\psi(w)-\psi(t))^{\rho}\right]\right|^{p} d t\right\} d w E_{\rho, \mu}^{\gamma}\left[\omega(\psi(w)-\psi(t))^{\rho}\right]\right|^{p} d t\right\} d w\right]^{\frac{1}{p}} \\
{\left[\int_{a}^{x}\left\{\int_{a}^{w}(\psi(w)-\psi(t))^{p(\mu-1)}\left|E_{\rho, \mu}^{\gamma}\left[\omega(\psi(w)-\psi(t))^{\rho}\right]\right|^{p} d t\right\} d w\right]^{\frac{1}{p}}} \\
\left(\int_{a}^{x}\left(\psi^{\prime}(w)|f(w)|\right)^{q} d w\right)^{\frac{1}{q}} \cdot
\end{gathered}
$$

The theorem is proved. 
The right side Opial inequality follows:

Theorem 2.6. Let $p, q>1: \frac{1}{p}+\frac{1}{q}=1$. Then

$$
\begin{gathered}
\int_{x}^{b}\left|\left(e_{\rho, \mu, \omega, b-}^{\gamma ; \psi} f\right)(w)\right||f(w)| \psi^{\prime}(w) d w \leq 2^{-\frac{1}{q}} \\
{\left[\int_{x}^{b}\left\{\int_{w}^{b}(\psi(t)-\psi(w))^{p(\mu-1)}\left|E_{\rho, \mu}^{\gamma}\left[\omega(\psi(t)-\psi(w))^{\rho}\right]\right|^{p} d t\right\} d w\right]^{\frac{1}{p}}} \\
\left(\int_{x}^{b}|f(w)|^{q}\left(\psi^{\prime}(w)\right)^{q} d w\right)^{\frac{2}{q}}
\end{gathered}
$$

$\forall x \in[a, b]$.

Proof. As it is similar to the proof of Theorem 2.5, is omitted.

We continue with more interesting Opial type Prabhakar-Caputo fractional inequalities:

Theorem 2.7. Let $p, q>1: \frac{1}{p}+\frac{1}{q}=1$. Then

i)

$$
\begin{gathered}
\int_{a}^{x}\left|\left({ }^{C} D_{\rho, \mu, \omega, a+}^{\gamma ; \psi} f\right)(w)\right|\left|f_{\psi}^{[N]}(w)\right| \psi^{\prime}(w) d w \leq 2^{-\frac{1}{q}} \\
{\left[\int_{a}^{x}\left\{\int_{a}^{w}(\psi(w)-\psi(t))^{p(N-\mu-1)}\left|E_{\rho, N-\mu}^{-\gamma}\left[\omega(\psi(w)-\psi(t))^{\rho}\right]\right|^{p} d t\right\} d w\right]^{\frac{1}{p}}} \\
\left(\int_{a}^{x}\left|f_{\psi}^{[N]}(w)\right|^{q}\left(\psi^{\prime}(w)\right)^{q} d w\right)^{\frac{2}{q}},
\end{gathered}
$$

and

ii)

$$
\begin{gathered}
\int_{x}^{b}\left|\left({ }^{C} D_{\rho, \mu, \omega, b-}^{\gamma ; \psi} f\right)(w)\right|\left|f_{\psi}^{[N]}(w)\right| \psi^{\prime}(w) d w \leq 2^{-\frac{1}{q}} \\
{\left[\int_{x}^{b}\left\{\int_{w}^{b}(\psi(t)-\psi(w))^{p(N-\mu-1)}\left|E_{\rho, N-\mu}^{-\gamma}\left[\omega(\psi(t)-\psi(w))^{\rho}\right]\right|^{p} d t\right\} d w\right]^{\frac{1}{p}}} \\
\left(\int_{x}^{b}\left|f_{\psi}^{[N]}(w)\right|^{q}\left(\psi^{\prime}(w)\right)^{q} d w\right)^{\frac{2}{q}},
\end{gathered}
$$

$\forall x \in[a, b]$.

Proof. By Theorems 2.5, 2.6 and (1.6)-(1.8).

Next come $\psi$-Hilfer-Prabhakar left and right Opial type fractional inequalities: 
Theorem 2.8. Let $p, q>1: \frac{1}{p}+\frac{1}{q}=1$. Additionally here assume that

$$
{ }^{R L} D_{\rho, \xi, \omega, a+}^{\gamma(1-\beta) ; \psi} f,{ }^{R L} D_{\rho, \xi, \omega, b-}^{\gamma(1-\beta) ; \psi} f \in C([a, b]) .
$$

Then

i)

$$
\begin{gathered}
\int_{a}^{x}\left|\left({ }^{H} \mathbb{D}_{\rho, \mu, \omega, a+}^{\gamma, \beta ; \psi} f\right)(w)\right|\left|\left({ }^{R L} D_{\rho, \xi, \omega, a+}^{\gamma(1-\beta) ; \psi} f\right)(w)\right| \psi^{\prime}(w) d w \leq 2^{-\frac{1}{q}} \\
{\left[\int_{a}^{x}\left\{\int_{a}^{w}(\psi(w)-\psi(t))^{p(\xi-\mu-1)}\left|E_{\rho, \xi-\mu}^{-\gamma \beta}\left[\omega(\psi(w)-\psi(t))^{\rho}\right]\right|^{p} d t\right\} d w\right]^{\frac{1}{p}}} \\
\left(\int_{a}^{x}\left|\left({ }^{R L} D_{\rho, \xi, \omega, a+}^{\gamma(1-\beta) ; \psi} f\right)(w)\right|^{q}\left(\psi^{\prime}(w)\right)^{q} d w\right)^{\frac{2}{q}}
\end{gathered}
$$

and

ii)

$$
\begin{gathered}
\int_{x}^{b}\left|\left({ }^{H} \mathbb{D}_{\rho, \mu, \omega, b-}^{\gamma, \beta ; \psi} f\right)(w)\right|\left|\left({ }^{R L} D_{\rho, \xi, \omega, b-}^{\gamma(1-\beta) ; \psi} f\right)(w)\right| \psi^{\prime}(w) d w \leq 2^{-\frac{1}{q}} \\
{\left[\int_{x}^{b}\left\{\int_{w}^{b}(\psi(t)-\psi(w))^{p(\xi-\mu-1)}\left|E_{\rho, \xi-\mu}^{-\gamma \beta}\left[\omega(\psi(t)-\psi(w))^{\rho}\right]\right|^{p} d t\right\} d w\right]^{\frac{1}{p}}} \\
\left(\int_{x}^{b}\left|\left({ }^{R L} D_{\rho, \xi, \omega, b-}^{\gamma(1-\beta) ; \psi} f\right)(w)\right|^{q}\left(\psi^{\prime}(w)\right)^{q} d w\right)^{\frac{2}{q}},
\end{gathered}
$$

$\forall x \in[a, b]$.

Proof. By Theorems 2.5, 2.6 and (1.15), (1.16).

Next we give several Prabhakar Hilbert-Pachpatte fractional inequalities. We start with a left side one.

Theorem 2.9. Let $p, q>1: \frac{1}{p}+\frac{1}{q}=1 ; i=1,2$. Let $\left[a_{i}, b_{i}\right] \subset \mathbb{R}, \psi_{i} \in C^{1}\left(\left[a_{i}, b_{i}\right]\right)$ and strictly increasing, $f_{i} \in C\left(\left[a_{i}, b_{i}\right]\right) ; \rho_{i}, \mu_{i}>0, \gamma_{i}, \omega_{i} \in \mathbb{R}$. Then

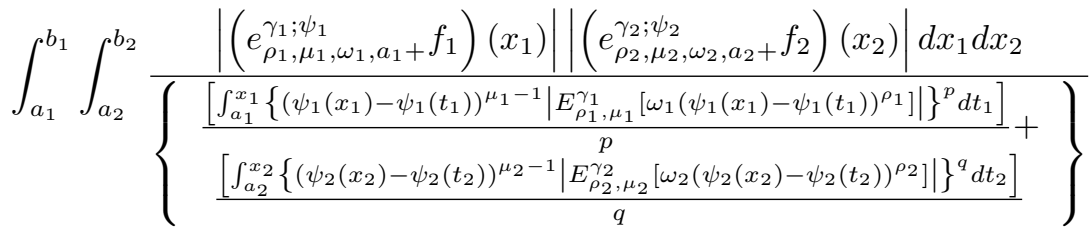

$$
\begin{aligned}
& \leq\left(b_{1}-a_{1}\right)\left(b_{2}-a_{2}\right)\left\|\psi_{1}^{\prime} f_{1}\right\|_{q}\left\|\psi_{2}^{\prime} f_{2}\right\|_{p} .
\end{aligned}
$$

Proof. We have that $(i=1,2)$

$$
\begin{gathered}
\left(e_{\rho_{i}, \mu_{i}, \omega_{i}, a_{i}+}^{\gamma_{i} \psi_{i}}\right)\left(x_{i}\right) \stackrel{(1.2)}{=} \\
\int_{a_{i}}^{x_{i}} \psi_{i}^{\prime}\left(t_{i}\right)\left(\psi_{i}\left(x_{i}\right)-\psi_{i}\left(t_{i}\right)\right)^{\mu_{i}-1} E_{\rho_{i}, \mu_{i}}^{\gamma_{i}}\left[\omega_{i}\left(\psi_{i}\left(x_{i}\right)-\psi_{i}\left(t_{i}\right)\right)^{\rho_{i}}\right] f_{i}\left(t_{i}\right) d t_{i},
\end{gathered}
$$


$\forall x_{i} \in\left[a_{i}, b_{i}\right]$, where $\rho_{i}, \mu_{i}>0 ; \gamma_{i}, \omega_{i} \in \mathbb{R}$.

Then

$$
\begin{gathered}
\left|\left(e_{\rho_{i}, \mu_{i}, \omega_{i}, a_{i}+}^{\gamma_{i} ; \psi_{i}} f_{i}\right)\left(x_{i}\right)\right| \leq \\
\int_{a_{i}}^{x_{i}} \psi_{i}^{\prime}\left(t_{i}\right)\left(\psi_{i}\left(x_{i}\right)-\psi_{i}\left(t_{i}\right)\right)^{\mu_{i}-1}\left|E_{\rho_{i}, \mu_{i}}^{\gamma_{i}}\left[\omega_{i}\left(\psi_{i}\left(x_{i}\right)-\psi_{i}\left(t_{i}\right)\right)^{\rho_{i}}\right]\right|\left|f_{i}\left(t_{i}\right)\right| d t_{i},
\end{gathered}
$$

$i=1,2, \forall x_{i} \in\left[a_{i}, b_{i}\right]$.

By appying Hölder's inequality twice we get:

$$
\begin{gathered}
\left|\left(e_{\rho_{1}, \mu_{1}, \omega_{1}, a_{1}+}^{\gamma_{1}, \psi_{1}} f_{1}\right)\left(x_{1}\right)\right| \leq \\
{\left[\int_{a_{1}}^{x_{1}}\left\{\left(\psi_{1}\left(x_{1}\right)-\psi_{1}\left(t_{1}\right)\right)^{\mu_{1}-1}\left|E_{\rho_{1}, \mu_{1}}^{\gamma_{1}}\left[\omega_{1}\left(\psi_{1}\left(x_{1}\right)-\psi_{1}\left(t_{1}\right)\right)^{\rho_{1}}\right]\right|\right\}^{p} d t_{1}\right]^{\frac{1}{p}}} \\
\left(\int_{a_{1}}^{x_{1}}\left(\psi_{1}^{\prime}\left(t_{1}\right)\left|f_{1}\left(t_{1}\right)\right|\right)^{q} d t_{1}\right)^{\frac{1}{q}}
\end{gathered}
$$

$\forall x_{1} \in\left[a_{1}, b_{1}\right]$, and

$$
\begin{gathered}
\left|\left(e_{\rho_{2}, \mu_{2}, \omega_{2}, a_{2}+}^{\gamma_{2} ; \psi_{2}} f_{2}\right)\left(x_{2}\right)\right| \leq \\
{\left[\int_{a_{2}}^{x_{2}}\left\{\left(\psi_{2}\left(x_{2}\right)-\psi_{2}\left(t_{2}\right)\right)^{\mu_{2}-1}\left|E_{\rho_{2}, \mu_{2}}^{\gamma_{2}}\left[\omega_{2}\left(\psi_{2}\left(x_{2}\right)-\psi_{2}\left(t_{2}\right)\right)^{\rho_{2}}\right]\right|\right\}^{q} d t_{2}\right]^{\frac{1}{q}}} \\
\left(\int_{a_{2}}^{x_{2}}\left(\psi_{2}^{\prime}\left(t_{2}\right)\left|f_{2}\left(t_{2}\right)\right|\right)^{p} d t_{2}\right)^{\frac{1}{p}}
\end{gathered}
$$

$\forall x_{2} \in\left[a_{2}, b_{2}\right]$.

Hence we have (by (2.28), (2.29))

$$
\begin{gathered}
\left|\left(e_{\rho_{1}, \mu_{1}, \omega_{1}, a_{1}+}^{\gamma_{1} ; \psi_{1}} f_{1}\right)\left(x_{1}\right)\right|\left|\left(e_{\rho_{2}, \mu_{2}, \omega_{2}, a_{2}+}^{\gamma_{2} ; \psi_{2}}\right)\left(x_{2}\right)\right| \leq \\
{\left[\int_{a_{1}}^{x_{1}}\left\{\left(\psi_{1}\left(x_{1}\right)-\psi_{1}\left(t_{1}\right)\right)^{\mu_{1}-1}\left|E_{\rho_{1}, \mu_{1}}^{\gamma_{1}}\left[\omega_{1}\left(\psi_{1}\left(x_{1}\right)-\psi_{1}\left(t_{1}\right)\right)^{\rho_{1}}\right]\right|\right\}^{p} d t_{1}\right]^{\frac{1}{p}}} \\
{\left[\int_{a_{2}}^{x_{2}}\left\{\left(\psi_{2}\left(x_{2}\right)-\psi_{2}\left(t_{2}\right)\right)^{\mu_{2}-1}\left|E_{\rho_{2}, \mu_{2}}^{\gamma_{2}}\left[\omega_{2}\left(\psi_{2}\left(x_{2}\right)-\psi_{2}\left(t_{2}\right)\right)^{\rho_{2}}\right]\right|\right\}^{q} d t_{2}\right]^{\frac{1}{q}}} \\
\left\|\psi_{1}^{\prime} f_{1}\right\|_{q}\left\|\psi_{2}^{\prime} f_{2}\right\|_{p} \leq
\end{gathered}
$$

(using Young's inequality for $a, b \geq 0, a^{\frac{1}{p}} b^{\frac{1}{q}} \leq \frac{a}{p}+\frac{b}{q}$ )

$$
\begin{gathered}
\left\{\frac{\left[\int_{a_{1}}^{x_{1}}\left\{\left(\psi_{1}\left(x_{1}\right)-\psi_{1}\left(t_{1}\right)\right)^{\mu_{1}-1}\left|E_{\rho_{1}, \mu_{1}}^{\gamma_{1}}\left[\omega_{1}\left(\psi_{1}\left(x_{1}\right)-\psi_{1}\left(t_{1}\right)\right)^{\rho_{1}}\right]\right|\right\}^{p} d t_{1}\right]}{p}+\right. \\
\left.\frac{\left[\int_{a_{2}}^{x_{2}}\left\{\left(\psi_{2}\left(x_{2}\right)-\psi_{2}\left(t_{2}\right)\right)^{\mu_{2}-1}\left|E_{\rho_{2}, \mu_{2}}^{\gamma_{2}}\left[\omega_{2}\left(\psi_{2}\left(x_{2}\right)-\psi_{2}\left(t_{2}\right)\right)^{\rho_{2}}\right]\right|\right\}^{q} d t_{2}\right]}{q}\right\} \\
\left\|\psi_{1}^{\prime} f_{1}\right\|_{q}\left\|\psi_{2}^{\prime} f_{2}\right\|_{p}
\end{gathered}
$$


$\forall x_{i} \in\left[a_{i}, b_{i}\right], i=1,2$.

So far we have

$$
\frac{\left|\left(e_{\rho_{1}, \mu_{1}, \omega_{1}, a_{1}+}^{\gamma_{1} ; \psi_{1}} f_{1}\right)\left(x_{1}\right)\right|\left|\left(e_{\rho_{2}, \mu_{2}, \omega_{2}, a_{2}+}^{\gamma_{2} ; \psi_{2}}\right)\left(x_{2}\right)\right|}{\frac{\left[\int_{a_{1}}^{x_{1}}\left\{\left(\psi_{1}\left(x_{1}\right)-\psi_{1}\left(t_{1}\right)\right)^{\mu_{1}-1}\left|E_{\rho_{1}, \mu_{1}}^{\gamma_{1}}\left[\omega_{1}\left(\psi_{1}\left(x_{1}\right)-\psi_{1}\left(t_{1}\right)\right)^{\rho_{1}}\right]\right|\right\}^{p} d t_{1}\right]}{p}+} \leq\left\|\psi_{1}^{\prime} f_{1}\right\|_{q}\left\|\psi_{2}^{\prime} f_{2}\right\|_{p},
$$

$\forall x_{i} \in\left[a_{i}, b_{i}\right], i=1,2$.

The denominator in (2.31) can be zero only when $x_{1}=a_{1}$ and $x_{2}=a_{2}$. Therefore we obtain (2.25) by integrating (2.31) over $\left[a_{1}, b_{1}\right] \times\left[a_{2}, b_{2}\right]$.

It follows the corresponding to (2.25) right side inequality.

Theorem 2.10. All as in Theorem 2.9. Then

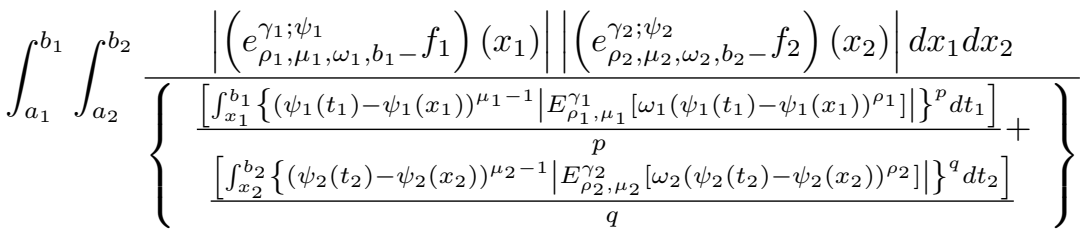

$$
\begin{aligned}
& \leq\left(b_{1}-a_{1}\right)\left(b_{2}-a_{2}\right)\left\|\psi_{1}^{\prime} f_{1}\right\|_{q}\left\|\psi_{2}^{\prime} f_{2}\right\|_{p} .
\end{aligned}
$$

Proof. As similar to the proof of Theorem 2.9 is omitted.

We continue with applications of Theorems 2.9, 2.10.

Theorem 2.11. Let $p, q>1: \frac{1}{p}+\frac{1}{q}=1 ; i=1,2$. Let $\left[a_{i}, b_{i}\right] \subset \mathbb{R}, \psi_{i} \in C^{\max \left(N_{1}, N_{2}\right)}\left(\left[a_{i}, b_{i}\right]\right)$, $\psi_{i}^{\prime} \neq 0$, and strictly increasing; $f_{i} \in C^{N_{i}}\left(\left[a_{i}, b_{i}\right]\right)$, where $N_{i}=\left\lceil\mu_{i}\right\rceil, 0<\mu_{i} \notin \mathbb{N}$. Here $\rho_{i}>0$; $\gamma_{i}, \omega_{i} \in \mathbb{R}$. Then

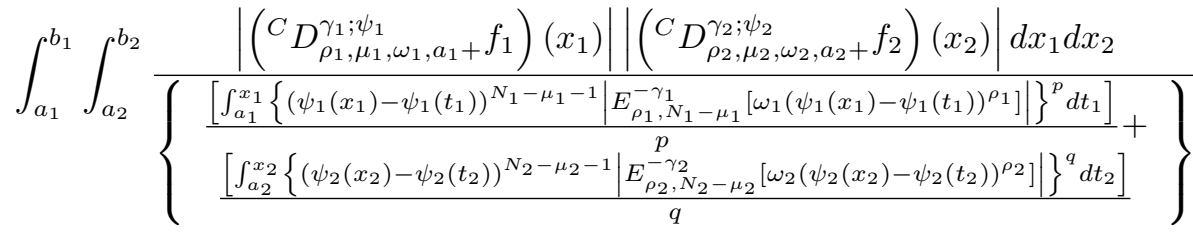

$$
\begin{aligned}
& \leq\left(b_{1}-a_{1}\right)\left(b_{2}-a_{2}\right)\left\|\psi_{1}^{\prime} f_{1 \psi_{1}}^{\left[N_{1}\right]}\right\|_{q}\left\|\psi_{2}^{\prime} f_{2 \psi_{2}}^{\left[N_{2}\right]}\right\|_{p} .
\end{aligned}
$$

Proof. By Theorem 2.9 and (1.2), (1.6).

We also give 
Theorem 2.12. All as in Theorem 2.11. Then

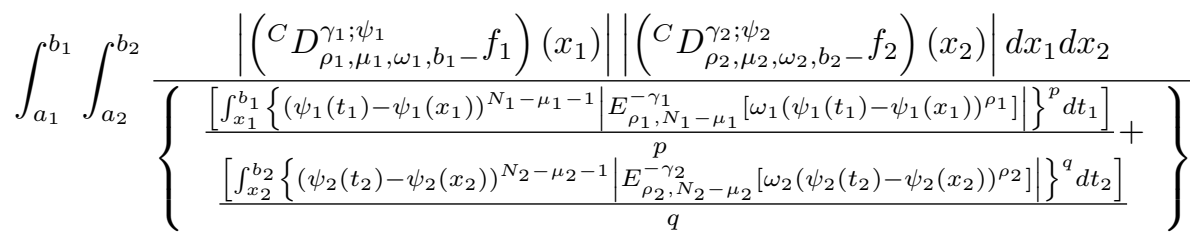

$$
\begin{aligned}
& \leq\left(b_{1}-a_{1}\right)\left(b_{2}-a_{2}\right)\left\|\psi_{1}^{\prime} f_{1 \psi_{1}}^{\left[N_{1}\right]}\right\|_{q}\left\|\psi_{2}^{\prime} f_{2 \psi_{2}}^{\left[N_{2}\right]}\right\|_{p} .
\end{aligned}
$$

Proof. By Theorem 2.10 and (1.3), (1.7).

We present

Theorem 2.13. Let $p, q>1: \frac{1}{p}+\frac{1}{q}=1 ; i=1,2$. Let $\left[a_{i}, b_{i}\right] \subset \mathbb{R}, \psi_{i} \in C^{\max \left(N_{1}, N_{2}\right)}\left(\left[a_{i}, b_{i}\right]\right)$, $\psi_{i}^{\prime} \neq 0$, and strictly increasing; $f_{i} \in C^{N_{i}}\left(\left[a_{i}, b_{i}\right]\right)$, where $N_{i}=\left\lceil\mu_{i}\right\rceil, 0<\mu_{i} \notin \mathbb{N}$. Here $\rho_{i}>0$; $\gamma_{i}, \omega_{i} \in \mathbb{R}$ and $\xi_{i}=\mu_{i}+\beta_{i}\left(N_{i}-\mu_{i}\right), i=1,2$, where $0 \leq \beta_{i} \leq 1$. Then

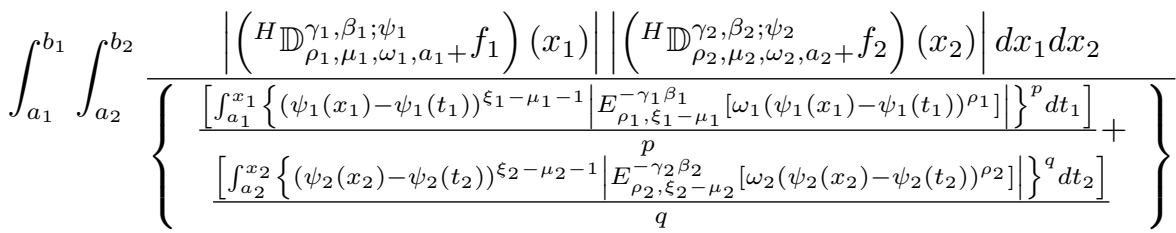

$$
\begin{aligned}
& \leq\left(b_{1}-a_{1}\right)\left(b_{2}-a_{2}\right)\left\|\psi_{1}^{\prime} R L D_{\rho_{1}, \xi_{1}, \omega_{1}, a_{1}+}^{\gamma_{1}\left(1-\beta_{1}\right) ; \psi_{1}} f_{1}\right\|_{q}\left\|\psi_{2}^{\prime} R L D_{\rho_{2}, \xi_{2}, \omega_{2}, a_{2}+}^{\gamma_{2}\left(1-\beta_{2}\right) ; \psi_{2}} f_{2}\right\|_{p} .
\end{aligned}
$$

Proof. By Theorem 2.9 and (1.15).

We also give

Theorem 2.14. All as in Theorem 2.13. Then

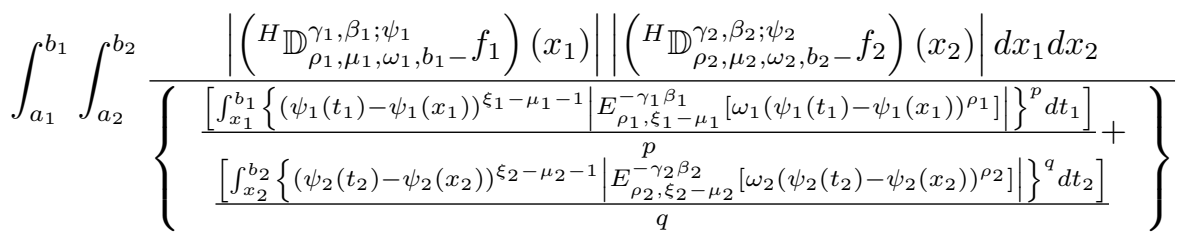

$$
\begin{aligned}
& \leq\left(b_{1}-a_{1}\right)\left(b_{2}-a_{2}\right)\left\|\psi_{1}^{\prime} R L D_{\rho_{1}, \xi_{1}, \omega_{1}, b_{1}-}^{\gamma_{1}\left(1-\beta_{1}\right) ; \psi_{1}} f_{1}\right\|_{q}\left\|\psi_{2}^{\prime}{ }^{R L} D_{\rho_{2}, \xi_{2}, \omega_{2}, b_{2}-}^{\gamma_{2}\left(1-\beta_{2}\right) ; \psi_{2}} f_{2}\right\|_{p} .
\end{aligned}
$$

Proof. By Theorem 2.10 and (1.16). 


\section{Appendix}

We give the following important fundamental results:

Theorem 3.1. Let $\rho, \mu>0 ; \gamma, \omega \in \mathbb{R} ;$ and $\psi \in C^{1}([a, b])$ increasing, $f \in C([a, b])$. Then $\left(e_{\rho, \mu, \omega, a+}^{\gamma ; \psi} f\right),\left(e_{\rho, \mu, \omega, b-}^{\gamma ; \psi} f\right) \in C([a, b])$.

Proof. We only prove $\left(e_{\rho, \mu, \omega, a+}^{\gamma ; \psi} f\right) \in C([a, b])$. We skip the proof for the other is similar.

We consider the power series

$$
\bar{E}_{\rho, \mu}^{\gamma}(z)=\sum_{k=0}^{\infty} \frac{\left|(\gamma)_{k}\right|}{k ! \Gamma(\rho k+\mu)(\rho k+\mu)} z^{k}, \quad z \in \mathbb{R} .
$$

We form

$$
\begin{gathered}
\bar{R}^{-1}:=\lim _{k \rightarrow \infty} \frac{\frac{\left|(\gamma)_{k+1}\right|}{(k+1) ! \Gamma(\rho(k+1)+\mu)(\rho(k+1)+\mu)}}{\frac{\left|(\gamma)_{k}\right|}{k ! \Gamma(\rho k+\mu)(\rho k+\mu)}}=\lim _{k \rightarrow \infty} \frac{\frac{|\gamma+k|}{(k+1) \Gamma(\rho(k+1)+\mu)(\rho(k+1)+\mu)}}{\frac{1}{\Gamma(\rho k+\mu)(\rho k+\mu)}}= \\
\lim _{k \rightarrow \infty} \frac{|\gamma+k| \Gamma(\rho k+\mu)(\rho k+\mu)}{(k+1) \Gamma(\rho(k+1)+\mu)(\rho(k+1)+\mu)}= \\
\lim _{k \rightarrow \infty}\left(\frac{|\gamma+k| \Gamma(\rho k+\mu)}{(k+1) \Gamma(\rho(k+1)+\mu)}\right) \lim _{k \rightarrow \infty}\left(\frac{\rho k+\mu}{(\rho k+\mu)+\rho}\right)=:(\Xi) .
\end{gathered}
$$

Notice that

$$
\lim _{k \rightarrow \infty}\left(\frac{\rho k+\mu}{(\rho k+\mu)+\rho}\right)=1
$$

From (1.1) we have that its radius of convergence is

$$
R=\lim _{k \rightarrow \infty} \frac{\frac{\left|(\gamma)_{k}\right|}{k ! \Gamma(\rho k+\mu)}}{\frac{\left|(\gamma)_{k+1}\right|}{(k+1) ! \Gamma(\rho(k+1)+\mu)}}=\lim _{k \rightarrow \infty} \frac{\frac{1}{\Gamma(\rho k+\mu)}}{\frac{|\gamma+k|}{(k+1) \Gamma(\rho(k+1)+\mu)}}=\lim _{k \rightarrow \infty} \frac{(k+1) \Gamma(\rho(k+1)+\mu)}{|\gamma+k| \Gamma(\rho k+\mu)}=\infty,
$$

because (1.1) is an entire function.

Therefore, we have that

$$
\lim _{k \rightarrow \infty} \frac{|\gamma+k| \Gamma(\rho k+\mu)}{(k+1) \Gamma(\rho(k+1)+\mu)}=0 .
$$

Consequently by (3.3), (3.4), we get that $(\Xi)=0$. Thus $\bar{R}^{-1}=0$ and the radius of convergence of $\bar{E}_{\rho, \mu}^{\gamma}(z)$, see (3.1), is $\bar{R}=\infty$, hence (3.1) is convergent everywhere.

Consequently it holds

$$
\sum_{k=0}^{\infty} \frac{\left|(\gamma)_{k}\right|\left(|\omega|(\psi(x)-\psi(a))^{\rho}\right)^{k}}{k ! \Gamma(\rho k+\mu)(\rho k+\mu)}<\infty
$$

$\forall x \in[a, b]$.

We notice that

$$
\sum_{k=0}^{\infty} \frac{\left|(\gamma)_{k}\right||\omega|^{k}}{k ! \Gamma(\rho k+\mu)} \int_{a}^{x} \psi^{\prime}(t)(\psi(x)-\psi(t))^{(\rho k+\mu)-1}|f(t)| d t \leq
$$




$$
\begin{gathered}
\|f\|_{\infty} \sum_{k=0}^{\infty} \frac{\left|(\gamma)_{k}\right||\omega|^{k}}{k ! \Gamma(\rho k+\mu)} \frac{(\psi(x)-\psi(a))^{\rho k+\mu}}{\rho k+\mu} \leq \\
\|f\|_{\infty}(\psi(b)-\psi(a))^{\mu} \sum_{k=0}^{\infty} \frac{\left|(\gamma)_{k}\right|\left(|\omega|(\psi(x)-\psi(a))^{\rho}\right)^{k}}{k ! \Gamma(\rho k+\mu)(\rho k+\mu)} \stackrel{(3.5)}{<} \infty .
\end{gathered}
$$

Consequently, by [5, p. 175$]$, we derive

$$
\begin{gathered}
\left(e_{\rho, \mu, \omega, a+}^{\gamma ; \psi} f\right)(x) \stackrel{(1.2)}{=} \int_{a}^{x} \psi^{\prime}(t)(\psi(x)-\psi(t))^{\mu-1}\left(\sum_{k=0}^{\infty} \frac{(\gamma)_{k}}{k ! \Gamma(\rho k+\mu)}\left(\omega(\psi(x)-\psi(t))^{\rho}\right)^{k}\right) f(t) d t \\
=\sum_{k=0}^{\infty} \frac{(\gamma)_{k} \omega^{k}}{k ! \Gamma(\rho k+\mu)} \int_{a}^{x} \psi^{\prime}(t)(\psi(x)-\psi(t))^{(\rho k+\mu)-1} f(t) d t
\end{gathered}
$$

$\forall x \in[a, b]$.

By [2, p. 98], we obtain that the function

$$
\lambda_{\rho, \mu}^{(k)}(f, x)=\int_{a}^{x} \psi^{\prime}(t)(\psi(x)-\psi(t))^{(\rho k+\mu)-1} f(t) d t
$$

$x \in[a, b]$, is absolutely continuous for $\rho k+\mu \geq 1$ and continuous for $\rho k+\mu \in(0,1) ; \psi \in C^{1}([a, b])$ and increasing.

That is always $\lambda_{\rho, \mu}^{(k)}(|f|, x) \in C([a, b])$, for all $k=0,1, \ldots$

By (3.5), one can derive that

$$
\begin{gathered}
\sum_{k=0}^{\infty} \frac{\left|(\gamma)_{k}\right||\omega|^{k}}{k ! \Gamma(\rho k+\mu)} \lambda_{\rho, \mu}^{(k)}(|f|, x) \leq \\
\|f\|_{\infty}(\psi(b)-\psi(a))^{\mu} \sum_{k=0}^{\infty} \frac{\left|(\gamma)_{k}\right|\left(|\omega|(\psi(b)-\psi(a))^{\rho}\right)^{k}}{k ! \Gamma(\rho k+\mu)(\rho k+\mu)}<\infty .
\end{gathered}
$$

Notice that

$$
\begin{aligned}
\left|\lambda_{\rho, \mu}^{(k)}(f, x)\right| \leq \lambda_{\rho, \mu}^{(k)}(|f|, x) & =\int_{a}^{x} \psi^{\prime}(t)(\psi(x)-\psi(t))^{(\rho k+\mu)-1}|f(t)| d t \\
& \leq\|f\|_{\infty} \frac{(\psi(b)-\psi(a))^{(\rho k+\mu)}}{(\rho k+\mu)}, k=0,1, \ldots
\end{aligned}
$$

And even more we get:

$$
\begin{gathered}
\frac{\left|(\gamma)_{k}\right||\omega|^{k}}{k ! \Gamma(\rho k+\mu)}\left|\lambda_{\rho, \mu}^{(k)}(f, x)\right| \leq \frac{\left|(\gamma)_{k}\right||\omega|^{k}}{k ! \Gamma(\rho k+\mu)} \lambda_{\rho, \mu}^{(k)}(|f|, x) \leq \\
\left(\frac{\left|(\gamma)_{k}\right||\omega|^{k}}{k ! \Gamma(\rho k+\mu)}\right) \frac{\|f\|_{\infty}(\psi(b)-\psi(a))^{(\rho k+\mu)}}{(\rho k+\mu)}=: M_{k}, \quad k=0,1, \ldots ;
\end{gathered}
$$

and by (3.8) that $\sum_{k=0}^{\infty} M_{k}<\infty$, converges.

By Weierstrass $M$-test we get that $\sum_{k=0}^{\infty} \frac{(\gamma)_{k} \omega^{k}}{k ! \Gamma(\rho k+\mu)} \lambda_{\rho, \mu}^{(k)}(f, x)$ is uniformly and absolutely convergent for $x \in[a, b]$.

Consequently by (3.7) we derive that $\left(e_{\rho, \mu, \omega, a+}^{\gamma ; \psi} f\right) \in C([a, b])$. The proof is completed. 
We finish with

Corollary 3.2. All as in Theorem 3.1. We have that

$$
\left\|e_{\rho, \mu, \omega, a+(b-)}^{\gamma ; \psi} f\right\|_{\infty} \leq\left(\sum_{k=0}^{\infty} \frac{\left|(\gamma)_{k}\right||\omega|^{k}(\psi(b)-\psi(a))^{\rho k+\mu}}{k ! \Gamma(\rho k+\mu+1)}\right)\|f\|_{\infty}<+\infty .
$$

That is $e_{\rho, \mu, \omega, a+(b-)}^{\gamma ; \psi}$ are bounded linear operators and positive operators if $\gamma, \omega>0$.

Proof. By (3.7), (3.8). 


\section{References}

[1] G. A. Anastassiou, Fractional differentiation inequalities, New York: Springer-Verlag, 2009.

[2] G. A. Anastassiou, Intelligent Computations: abstract fractional calculus inequalities, approximations, Cham: Springer, 2018.

[3] A. Giusti, I. Colombaro, R. Garra, R. Garrappa, F. Polito, M. Popolizio and F. Mainardi, "A practical guide to Prabhakar fractional calculus", Fract. Calc. Appl. Anal., vol. 23, no. 1, pp. 9-54, 2020.

[4] R. Gorenflo, A. Kilbas, F. Mainardi and S. Rogosin, Mittag-Leffler functions, related topics and applications, Heidelberg: Springer, 2014.

[5] E. Hewith and K. Stromberg, Real and abstract analysis. A modern treatment of the theory of functions of a real variable, New York: Springer, 1965.

[6] F. Polito and Ž. Tomovski, "Some properties of Prabhakar-type fractional calculus operators", Fract. Differ. Calc., vol. 6, no. 1, pp. 73-94, 2016.

[7] T. R. Prabhakar, "A singular integral equation with a generalized Mittag-Leffler function in the kernel", Yokohama Math. J., vol. 19, pp. 7-15, 1971.

[8] J. Vanterler da C. Sousa, E. Capelas de Oliveira, "On the $\psi$-Hilfer fractional derivative", Commun. Nonlinear Sci. Numer. Simul., vol. 60, pp. 72-91, 2018. 